\title{
Applying parameter identification and optimal input design in well-testing
}

\author{
BJARNE A. FOSS
}

Keywords: reservoir, well-testing, parameter identification, optimal input design.

\begin{abstract}
This article discusses the use of optimal input design and parameter identification methods in reservoir evaluation. A new optimal input design criterion is presented which in addition to the uncertainty in the parameter estimates accounts for the curvature of the parameter space.

It is shown that the information content in the data obtained from a well-test generally increases markedly, by actively using the optimal input design method. The use of parameter identification (calculating error levels) improves the extraction of information from reservoir data, since the inclusion of prior knowledge and the parameterization level may be chosen according to acceptable uncertainties in the parameter estimates.
\end{abstract}

\section{Introduction}

The process of characterizing the properties of a hydrocarbon reservoir may be divided into several stages according to the data which is used.

Seismic exploration data is used to identify the macro-geological structure, for instance the layering of a certain formation as a basis for deciding if and where to drill wells. Having drilled a well, well logging is used to obtain data which characterizes the rock and fluid properties in the vicinity of a well, to a certain extent. In addition, laboratory analysis of reservoir rocks and fluids are carried out.

Well-testing is an important tool which is used to investigate reservoir conditions beyond a well. Such a test is performed by producing from one or several wells for a short time-period (typically 5-30 hours). This implies that only a small part of the reservoir will be influenced by the test, see Fig. 1. The flow-rate(s) and pressure(s) are always monitored during this time period.

A well-test will often have several objectives in addition to estimating reservoir properties (particularly the permeabilities). These may include obtaining a pressure profile vs. depth, collecting reservoir fluid samples, and measuring the sand production and the gas to oil production ratio as a function of the flow-rate(s).

During the production stages of a reservoir, data may be available from the different production (or injection) wells. This data may consist of flow-rates, water to oil production ratios, gas to oil production ratios and pressures. It is common practice today to use such data for history matching, i.e., manually adjusting parameters in a reservoir simulator in order to match the data to the simulator output.

The importance of obtaining a good reservoir model lies in its use for optimizing field development in order to improve the usage of hydrocarbon fields.

Received 14 May 1988.

† SINTEF, Division of Automatic Control, 7034 Trondheim, Norway. 


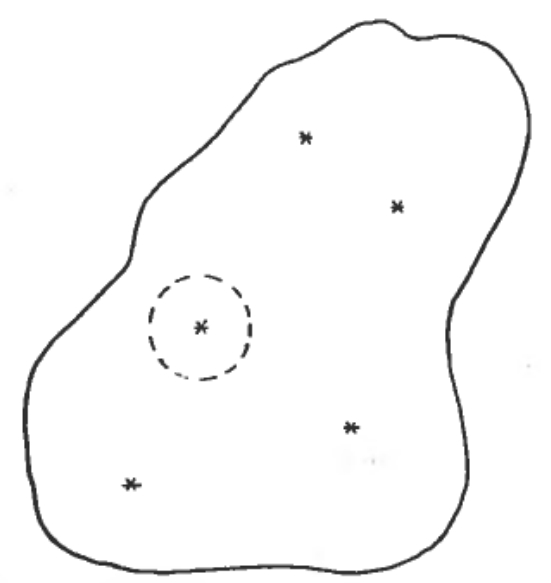

Figure 1. An areal view of a hydrocarbon reservoir with wells (*) showing the investigation area (dotted) of a typical single well well-test.

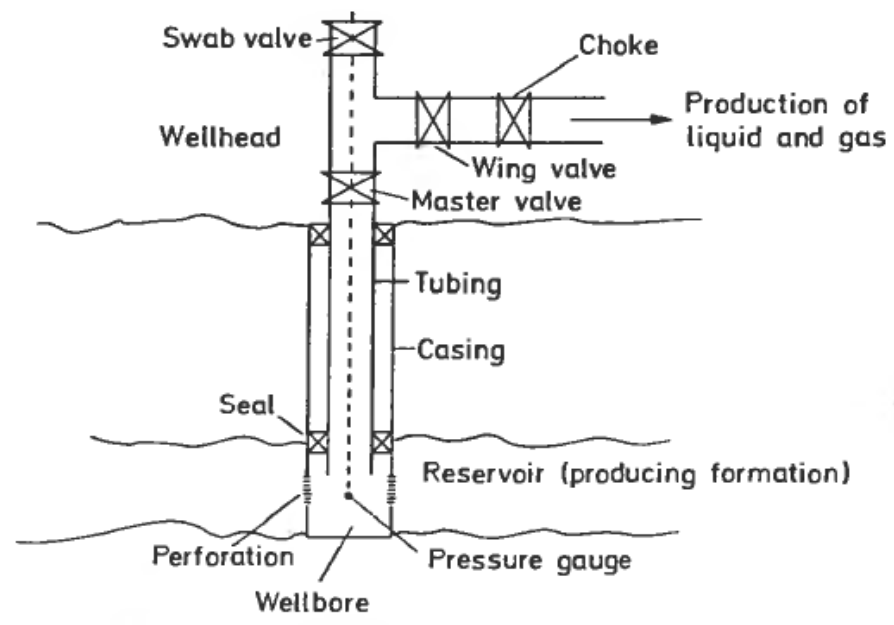

Figure 2. A schematic view of a well-test scenario.

The above discussion shows that there are several sources of information which are used to obtain a good understanding of a reservoir. In this study we will emphasize well-testing. A schematic well completion which is typical for a well-test is shown in Fig. 2. The flow-rate is adjusted by the choke value and monitored downstream the test separator (not shown in the figure).

Normally well-tests are analysed by the use of a graphical method, see Lee 1982 for example. This investigation aims at using parameter identification and optimal input design methods in well-testing.

In $\S 2$ an appropriate model is presented while $\S 3$ deals with methods for parameter identification and optimal input design. Results are presented in $\S 4$ while a discussion on the use of the methods in well-testing $(\$ 5)$ concludes this paper.

\section{Model}

\subsection{Reservoir model}

The reservoir to be considered is a single-well system, the main assumptions being 2-dimensional cylindrical geometry, see Fig. 3. and 1-phase flow. These assumptions are valid in many well-test situations. The geometric assumption dis- 


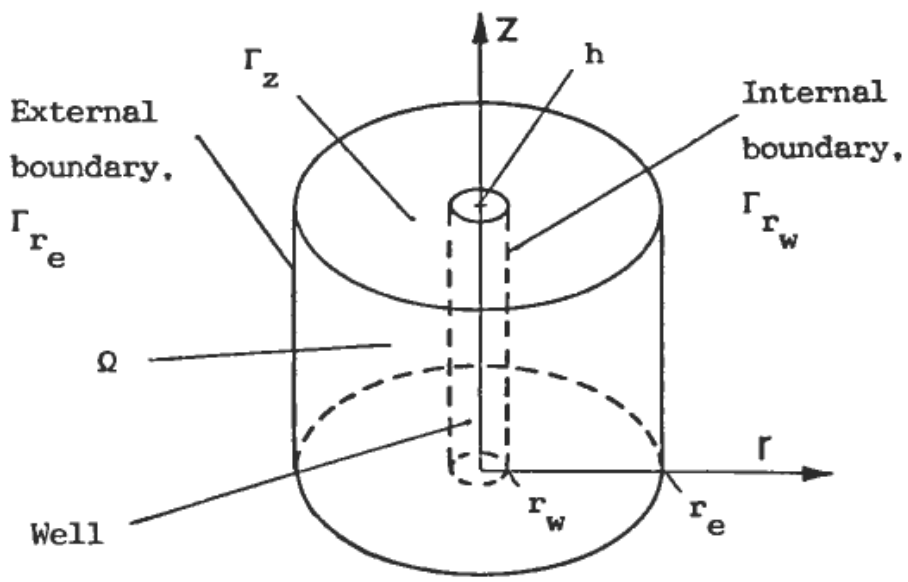

Figure 3. Model geometry.

regards reservoirs with significant variations about a 3rd coordinate axis. In undersaturated reservoirs and in gas wells the flow assumption is most often valid.

The equations governing the dynamics are developed several places in the petroleum literature, an example of this is Aziz and Settari (1979). The derivation uses mass balance in cylindrical coordinates, Darcy's law for flow in porous media and isothermal compressibility to obtain the following parabolic partial differential equation:

$$
\begin{gathered}
p=p(r, z, t), \quad k^{r}=k^{r}(r, z), \quad k^{z}=k^{z}(r, z), \quad \tau=\Phi \mu c \\
\tau \frac{\partial p}{\partial t}=\frac{1}{r} \frac{\partial}{\partial r}\left(k^{r} r \frac{\partial p}{\partial r}\right)+\frac{\partial}{\partial z}\left(k^{z}\left(\frac{\partial p}{\partial z}+\rho g\right)\right) \\
\text { in } \Omega \times(t>0), \quad \Omega=\left\langle r_{w}, r_{e}\right\rangle \times\langle 0, h\rangle
\end{gathered}
$$

Initial conditions

$$
p(r, z, 0)=p_{\text {init }}(r, z)=p_{\mathrm{o}}-\rho g z \text { in } \Omega \cup \Gamma
$$

Boundary conditions

$$
\begin{aligned}
& \frac{\partial p}{\partial z}+\rho g=0 \quad \text { on } \quad \Gamma_{z} \Lambda t>0 \\
& \frac{\partial p}{\partial r}=0 \quad \text { on } \quad \Gamma_{r_{e}} \Lambda t>0 \\
& \frac{\partial p}{\partial r}=\frac{q(z, t) \mu}{2 \pi r_{w} k^{r}\left(r_{w}, z\right)} \text { on } \Gamma_{r_{w}} \Lambda t>0
\end{aligned}
$$

where $\Omega$ is the reservoir interior and $\Gamma$ is the reservoir boundary (see Fig. 3). This model assumes fluid-flow, but can be used for gas wells by introducing the notion of 'real gas pseudo pressure'. This implies that an integral transform of the pressure is used instead of the pressure itself in the gas flow case, see Dake (1978), Chapter 8.

\subsection{Model discretization}

The continuous model in eqns. (1)-(5) is discretized using the finite-difference technique. An irregular grid is used, as shown in Fig. 4. In the case of a single phase (fluid-flow) problem with radial geometry and homogeneous rock properties the 


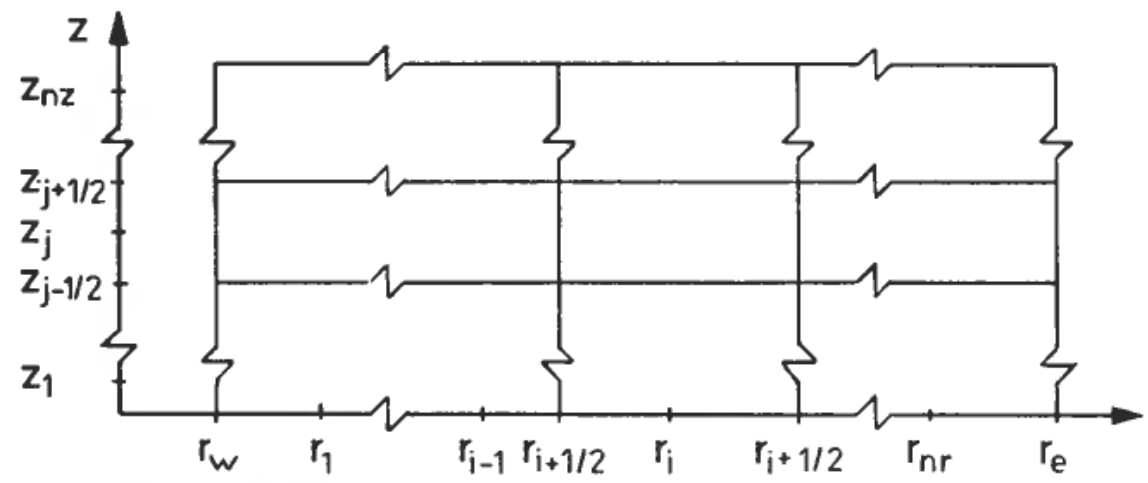

Figure 4. Spatial discretization of the reservoir. $i+1 / 2, j+1 / 2$ indicate grid boundaries.

stationary solution of the pressure increases linearly with $\ln r$. A suitable way in which to construct an irregular grid is therefore,

$$
\ln \frac{r_{i+1}}{r_{i}}=\ln \frac{r_{i}}{r_{i-1}}, \quad i=2, \ldots, n r-1
$$

In addition the grid-block boundaries $\left(r_{i+1 / 2}, i=1, \ldots, n r-1\right)$ must be chosen. This is, e.g., discussed in Aziz and Settari (1979), Chapter 3 and 7. The boundary values for calculating the block volume are given by:

$$
r_{1+1 / 2}=\sqrt{\left(\left(r_{i+1}^{2}-r_{i}^{2}\right) / \ln \left[\frac{r_{i+1}}{r_{i}}\right]^{2}\right)}
$$

This expression is derived by comparing the flow past the grid block boundary (for a given pressure difference) with the flow generated by integrating Darcy's law between the grid points in grid block $i$ and $i+1$.

In the vertical direction a block-centred grid is used, i.e., the block boundaries are chosen according to perforations and reservoir discontinuities, and the grid points are placed in the centres of the blocks. The time derivative is approximated by a backwards Euler approximation, resulting in the following linear, implicit discrete time model:

$$
A p^{n+1}=p^{n}+B p_{r j}^{n+1}, \quad n=1, \ldots, N-1
$$

The pressure drops from the grid point adjacent to the well $(i=1$, see Fig. 4$)$ to the corresponding pressure in the well is used as input in eqn. (8).

$$
p_{r j}^{n}=p_{1 j}^{n}-\left(p_{w}^{n}-\rho g z\right), \quad j=1, \ldots, n z
$$

An expression for $p_{r j}$ as a function of the production rate is derived by the use of a 1 st order approximation to the inner boundary condition, eqn. (5).

$$
p_{r j}^{n}=\frac{\left(r_{1}-r_{w}\right) / r_{w}}{2 \pi \Delta z_{j} k_{j}^{s}} q_{j}^{n}=\beta_{j} q_{j}^{n}, \quad j=1, \ldots, n z
$$

The permeability in a thin zone around the well, $k^{s}$, differs from the permeability further out, because of disruptions during the drilling and the completion of a well.

Usually the total flow-rate from a well is specified. If there is production from more than one layer, $p_{r j}$ depends on the reservoir pressure. In this case the well 
equations must be implicit in order to maintain the stability properties of the total model.

The simulator model is checked against the mass balance of the reservoir by using the compressibility relation,

$$
c=-\frac{1}{V_{p}} \frac{\partial V}{\partial p}=-\frac{1}{V_{p}} \frac{\partial V / \partial t}{\partial p / \partial t}
$$

where $V_{p}$ denotes the pore volume, $\partial V / \partial t$ the production rate and $\partial p / \partial t$ the average pressure decline.

\subsection{Parametrization}

Determining the reservoir permeabilities is of particular concern in well-testing since this is the only means of obtaining good estimates of these crucial parameters in the pre-production stages of a reservoir. The permeabilities are proportionality factors which relate fluid-flow rates to pressure gradients (Darcy's law). They vary as general functions of the spatial coordinates, hence they are infinite dimensional.

In the discretized model it is assumed that the spatially variable unknown parameters (in our examples the permeabilities) have a constant value within each grid block. This approach has, however, been combined extensively with zonation, i.e., assuming equal parameter values in several grid blocks so as to reduce the number of unknown parameters.

\section{Identification}

\subsection{Parameter estimation}

The optimal parameter estimates are computed by minimizing a quadratic criterion as shown below.

$$
J=\frac{1}{2} \sum_{n=1}^{N} \sum_{l=1}^{L} w_{l}\left(y_{l}^{n}-\hat{y}_{l}^{n}\right)^{2}, \quad \varepsilon_{l}^{n}=y_{l}^{n}-\hat{y}_{l}^{n}, \quad w_{l} \geqslant 0
$$

The minimization is performed using a quasi-Newton method with the following specific features:

A line search is used.

The Hessian matrix is scaled so as to improve its conditioning.

If the scaled Hessian matrix is badly conditioned a diagonal matrix with equal diagonal elements is added to the scaled Hessian matrix.

The gradient is computed by simulating the sensitivity equations, i.e., the equations which arise by differentiating eqns. (8) and (10) with respect to the unknown parameters.

\subsection{Error levels}

The analysis of the Hessian matrix, i.e. $\partial^{2} J / \partial \Theta \partial \Theta^{\prime}$, together with the noise in the observed data is an important tool when choosing the parameters which can be estimated in a given situation. The reason is that this gives a quantitative measure of the uncertainty associated with a parameter estimate. In general, it is better to fix 
the value of an unknown parameter instead of trying to estimate it with large error bounds. In addition, fixing one parameter will often reduce the uncertainty of the estimates of the other unknown parameters.

Given the following assumption,

stationary, additive uncorrelated noise on the data $\left(e_{l}\right.$ in eqn. (13))

a linear model

no modelling errors

it is possible to derive an expression of the variance of the unbiased parameter estimates, see Foss 1987(a), Appendix J.

$$
\begin{gathered}
\operatorname{Var}\left(\hat{\Theta}_{m}\right)=\max _{k=1, M}\left[\frac{v_{m k}^{2}}{\lambda_{k}(\boldsymbol{H}) M}\right] \cdot \sum_{t=1}^{L} w_{l} \operatorname{Var}\left(e_{l}\right), \quad m=1, \ldots, M \\
\boldsymbol{H}=\partial^{2} J / \partial \Theta \partial \Theta^{\prime} \approx \frac{1}{N} \sum_{n=1}^{N} \sum_{l=1}^{L}\left[\left(w_{l} \frac{\partial y_{l}^{n}}{\partial \Theta} \frac{\partial y_{l}^{n}}{\partial \Theta^{\prime}}\right)\right]
\end{gathered}
$$

This derivation is based on an eigenvalue decomposition of the Hessian matrix, $v_{m k}$ being elements of the eigenvector matrix and $\lambda_{k}$ the $k^{\prime}$ th eigenvalue. The Hessian matrix in eqn. (14) is computed by neglecting the 2 nd order term.

\subsection{Optimal input design}

In optimal input design theory it is common to assume that an unbiased, minimum variance estimator is used, hence it is just the variance that determines the uncertainty. The minimum variance (efficient) estimate can be calculated by the use of the Fisher information matrix, see Silvey (1970), pp. 35-44. Under suitable conditions, see Mehra (1974), the Fisher information matrix can be approximated using the Hessian matrix in eqn. (14). The input design method may now be written in a compact form

$$
\min _{q \in Q} g\left(\boldsymbol{H}^{-1}\right)
$$

by recognizing the fact that $\boldsymbol{H}$ directly influences the uncertainty of the parameter estimates, and that the input, $q$, to the system in question, influences $H$. $Q$ is the set of admissible inputs, $g$ is a functional. Several choices for $g$ choices have been proposed in the literature, see Mehra (1974). The one used in this investigation is based on an augmentation of the standard criteria. It was first presented in Foss (1987(b)). Below the commonly denoted $E$-optimality criterion (i.e., the use of the maximum eigenvalue of the inverse Hessian matrix) is augmented. This criterion is used in $\S 4$.

$$
g\left(\boldsymbol{H}^{-1}\right)=\lambda_{\max }\left(\boldsymbol{H}^{-1}\right)+\xi \kappa(\boldsymbol{H}), \quad \xi \geqslant 0
$$

$\lambda_{\max }$ is the maximum eigenvalue and $\kappa$ is the condition number. The first term places emphasis on reducing parameter uncertainty by reducing the largest eigenvalue of $\boldsymbol{H}^{-1}$ while the second term considers the curvature of the parameter space. It is important to keep the condition number as low as possible since this improves the robustness of the parameter estimation runs which folow the experiment. In the cases where $\lambda_{\max }\left(\boldsymbol{H}^{-1}\right)$ shows a flat minimum it is especially advantageous to incorporate the condition number in the criterion, see Foss $(1987(b))$ for a further discussion on this. 


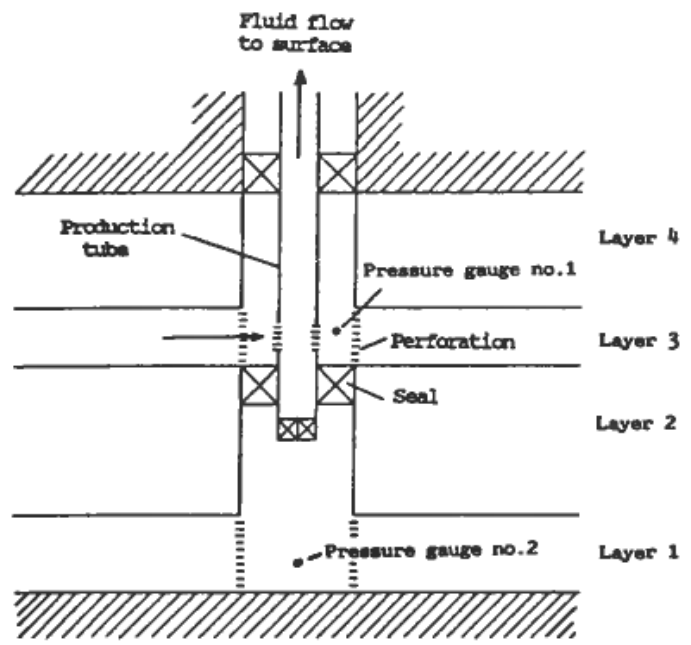

Figure 5. A schematic view of the production and monitoring equipment, and the reservoir layering.

\section{Results}

The outlined approach will be used in two different examples. First, a simulated realistic well-test situation is discussed with respect to parameter identification and optimal input design. Second, the data from a North Sea well-test is analysed.

\section{I. A simulated well-test example}

In this example we assume a reservoir geometry as shown in Fig. 3. The characteristic properties of the reservoir are shown in Table 1 while Fig. 5 gives a schematic view of the well and reservoir layering.

Both the 1st and 3rd layer are perforated, only the 3rd layer is in direct contact with the well however. The pressure is monitored in two locations $(L=2)$. Two different permeability distributions will be discussed as shown in Fig. 6. The permeability is generally directional, typically the vertical permeabilities have much lower values than the horizontal permeabilities.

The total well-testing time is chosen to be 28 hours with a sampling time of 250 secs. It is at first assumed that the well produces at a constant rate of $10 \mathrm{~m}^{3} /$ hour (starting at time zero) during the entire testing period.

A candidate parameter vector is shown below,

$$
\Theta^{\prime}=\left[k_{1}^{r} k_{2}^{r} k_{3}^{r} k_{2}^{z} k_{3}^{z} k_{4}^{z} k^{s}\right]
$$

while the loss function, defined in eqn. (12), uses $w_{1}=0.02, w_{2}=1.00$ and $N=400$.

\begin{tabular}{ll}
\hline Wellbore radius: $r_{w}=0.15 \mathrm{~m}$ & Exterior radius: $r_{e}=750 \cdot 0 \mathrm{~m}$ \\
Reservoir thickness: $h=75 \cdot 0 \mathrm{~m}$ & Porosity: $\Phi=0 \cdot 25$ \\
Viscosity: $\mu=10^{-3} \mathrm{Pas}=1 \mathrm{cP}$ & Compressibility: $c=10^{-9} \mathrm{~Pa}^{-1}$ \\
Initial pressure: $p_{\text {init }}=30 \mathrm{MPa} \simeq 4350 \mathrm{psi}$ \\
Pore volume: $33 \cdot 1310^{6} \mathrm{~m}^{3}$ or $20810^{6} \mathrm{bbl}$ Water saturation: $S_{w}=0$ \\
\hline
\end{tabular}

Table 1. Characteristic properties of the reservoir. 


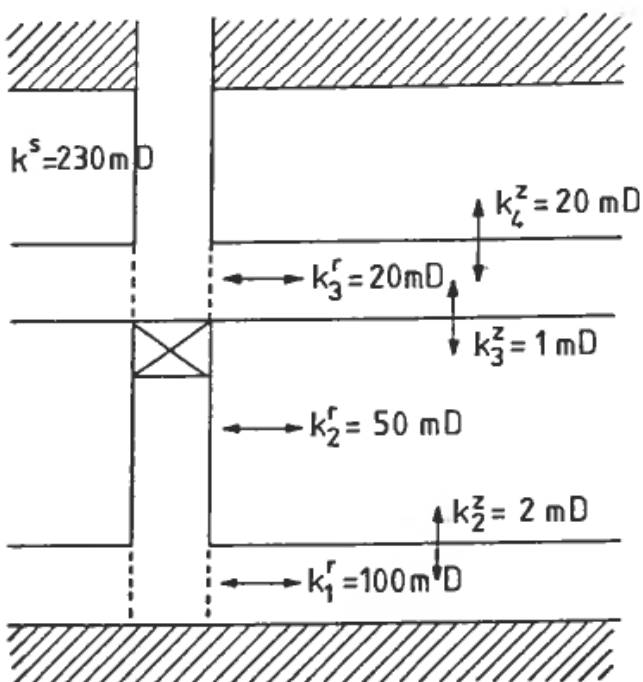

Case 1

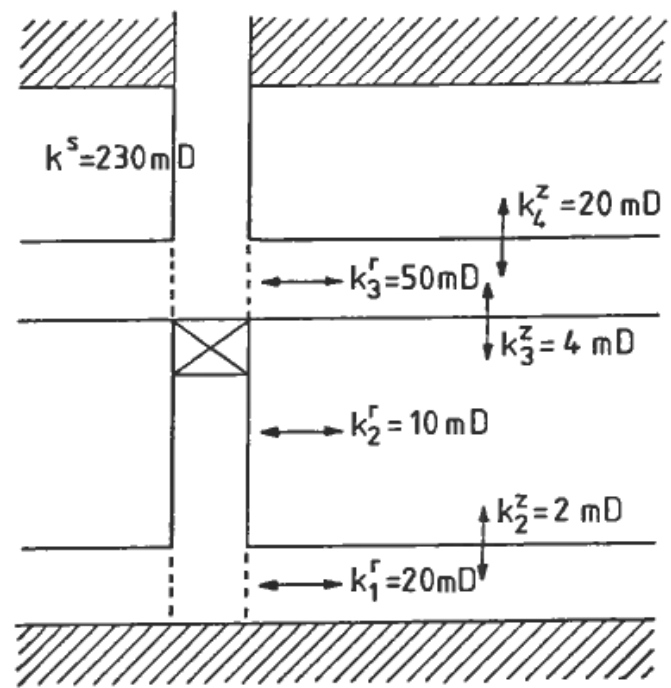

Case 2

Figure 6. Permeability distribution.

Case 1 and 2 in Figure 6 give rise to the following eigenvalues of the Hessian matrices

$$
\begin{aligned}
& \lambda_{1}(\boldsymbol{H})^{\prime}=\left[\begin{array}{lcccccc}
27115 & 25.2 & 11.5 & 0.91 & 0.35 & 1.0 \times 10^{-4} & 1.0 \times 10^{-5}
\end{array}\right] \\
& \lambda_{2}(\boldsymbol{H})^{\prime}=\left[\begin{array}{lllllll}
60325 & 2861 & 11.8 & 6.5 & 0.45 & 0.14 & 1.2 \times 10^{-3}
\end{array}\right]
\end{aligned}
$$

Both $\lambda_{1}$ and $\lambda_{2}$ are unacceptable because of the large uncertainty associated with the smallest eigenvalues. All the same, the results are important since they support an argument which should be taken into account before choosing the well completion for a test.

Case 1 and 2 are very different in that permeability decreases towards the producing perforation in Case 1 while the opposite may be said of Case 2. Intuitively this should decrease the identifiability in Case 1, since the area which has the lowest permeability close to the producing perforation will disguise the effect of pressure changes in high permeability areas further into the reservoir. This is an example of how an identifiability analysis can be helpful when designing the well completion for a test. In Case 1 for instance it is preferable (from an identifiability point of view) to produce from layer 1 instead of layer 3 . The permeability distribution is of course not known prior to the test, an indication of permeability variations from well logging and analysis of the reservoir rocks (from the drilling of the well) is however available in most cases.

Pursuing the identifiability analysis in Case 2, added knowledge must be utilized in order to reduce parameter confidence intervals. Analysis shows that identifiability suffers most from the fact that both $k_{1}^{r}$ and $k_{2}^{r}$ are estimated independently. If it can be assumed that these are correlated, for instance as follows,

$$
k_{1}^{r}=2 k_{2}^{r}
$$

the eigenvalues change to

$$
\lambda_{3}(\boldsymbol{H})^{\prime}=\left[\begin{array}{llllll}
61372 & 2943 & 40 \cdot 9 & 7 \cdot 4 & 0 \cdot 42 & 0 \cdot 14
\end{array}\right]
$$




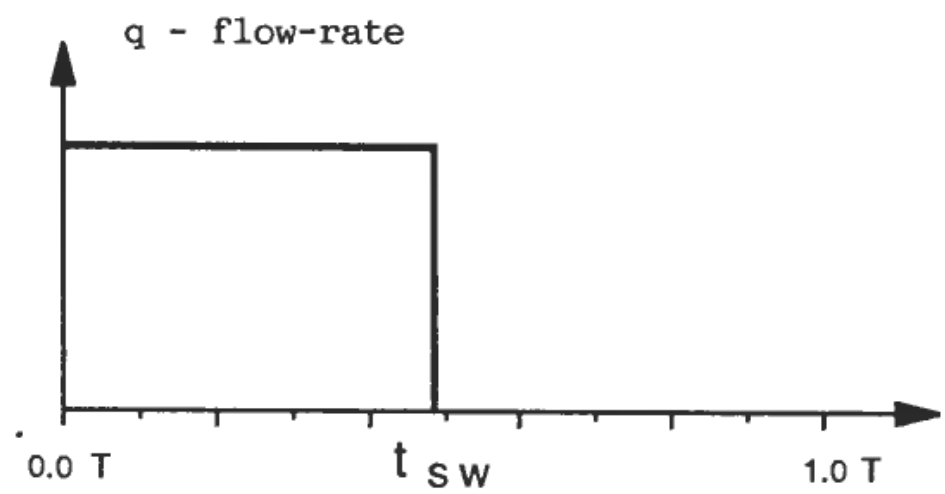

Figure 7. Restrictions on the production flow-rate, $q$, in the input design problem. $T$ is the total well-testing time, 28 hours.

A further reduction in confidence intervals is sought by looking at input design techniques. The choice of the flow-rate during the well-test is limited as given in Fig. 7. It is uniquely defined by choosing the switch-off time, $t_{s w}$. This is a common way of running a well-test. The results are shown in Fig. 8. It shows (open markers) that production should be terminated after only $7-25 \%$ of the total testing time. This result did not change significantly if other permeability values were chosen, within the same range of values as above.

Figure 8 highlights the use of the augmented input design criterion. The minimum of the two design criteria shown in Fig. 8 appear at very different switchoff time instants. For practical purposes the input sequence obtained by the augmented criterion is superior, since the condition number of the Hessian matrix is reduced by a factor of ten at the expense of a marginal increase in the largest eigenvalue.

In Foss $(1987(a))$, other examples illustrate the importance of considering the conditioning of the parameter space since a smoother parameter space improves the convergence rate and makes the optimization more robust, hence the parameter optimization will be more reliable in converging in non-ideal situations.

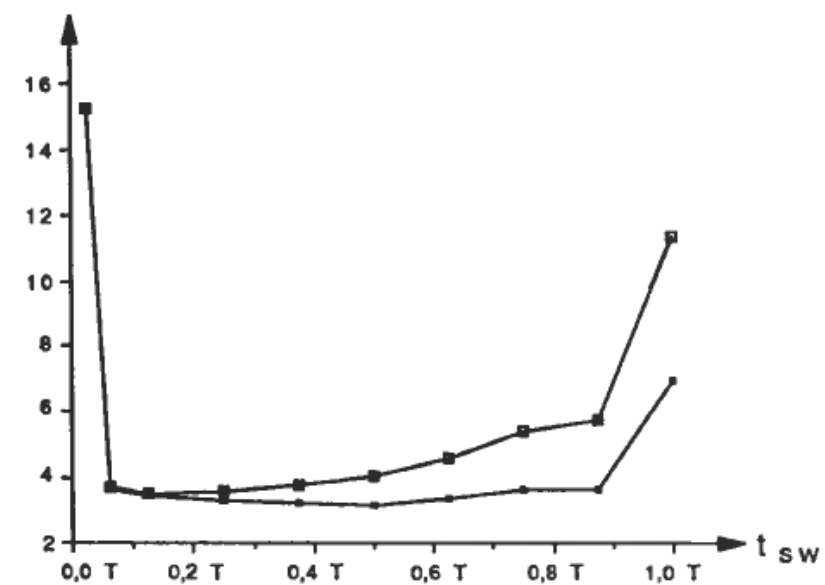

Figure 8. The value of the optimal input design criterion $\left(\xi=1.010^{-5}\right.$ open markers, $\xi=0$ filled markers) using $\Theta^{\prime}=\left[k_{2}^{r} k_{3}^{r} k_{2}^{z} k_{3}^{z} k_{4}^{z} k^{3}\right]$ for different switch-off time instants. The parameter values in Case 2, see Fig. 6, are used. 


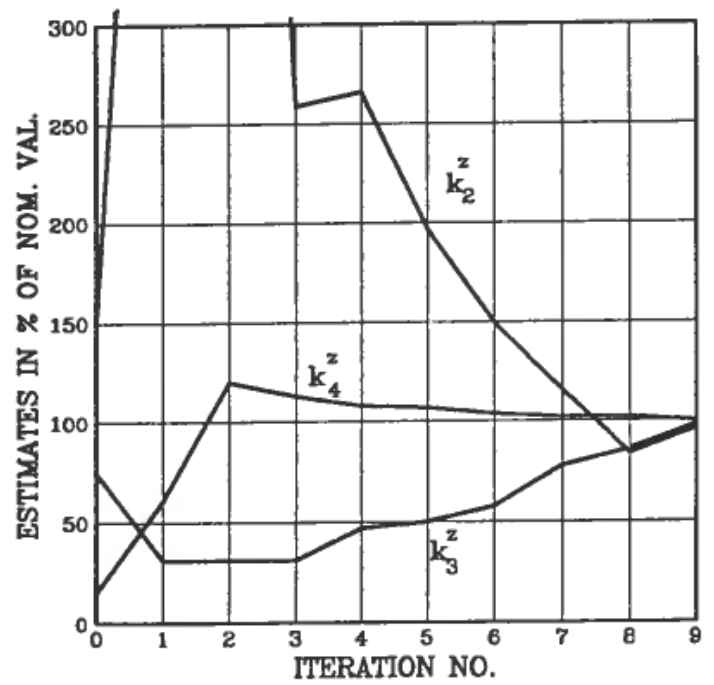

Figure 9. Parameter optimization using the input sequence defined by $t_{s w}=0 \cdot 125 T$. Initial parameter estimates

$\Theta^{\prime}=\left[k_{2}^{r} k_{3}^{r} k_{2}^{z} k_{3}^{z} k_{4}^{z} k^{r}\right]$

$=\left[\begin{array}{llllll}20 \mathrm{mD} & 100 \mathrm{mD} & 3 \mathrm{mD} & 3 \mathrm{mD} & 3 \mathrm{mD} & 250 \mathrm{mD}\end{array}\right]$

A typical successful parameter optimization run is shown in Fig. 9. The optimization run shows good performance, the other three estimates perform equally well. A corresponding run was also carried out using a constant production rate over the entire test period $\left(t_{s w}=1.00 \mathrm{~T}\right)$ to check the importance of the input design considerations. The run did not converge. Similar runs using smaller errors in the initial estimates did not converge either. The reason for this is the ill-conditioned parameter space which makes it extremely difficult to find the optimal parameter estimates.

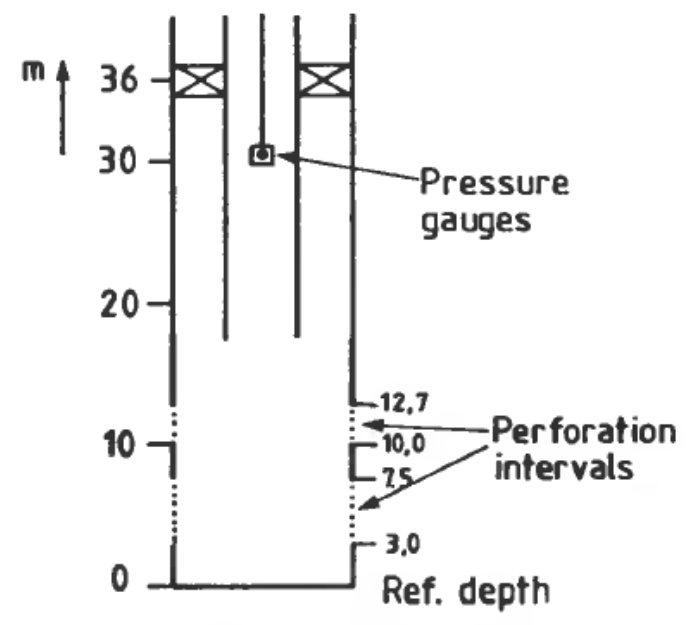

Figure 10. Well completion. 


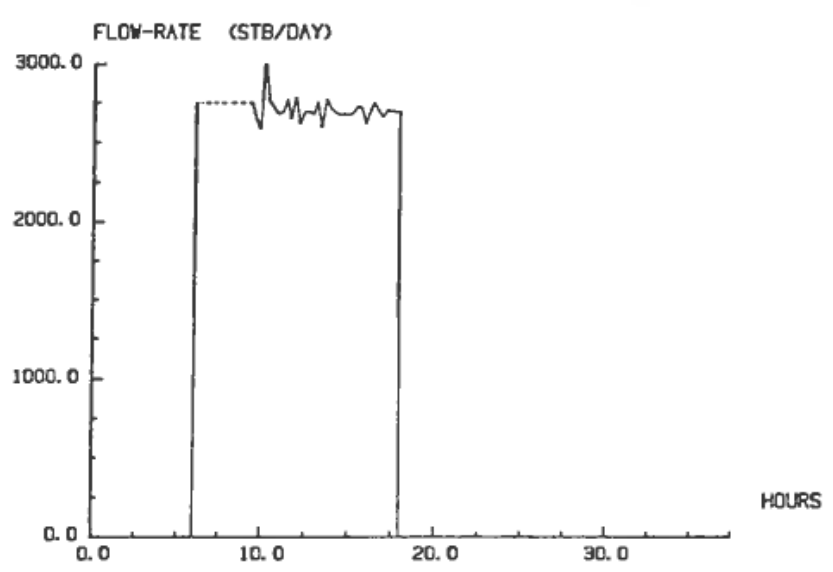

Figure 11. Flow-rate during the main flow period.

\subsection{Analysing data from a North Sea well-test}

This test stems from a well about $2500 \mathrm{~m}$ deep. Two intervals of oil bearing formation were perforated, see Fig. 10. Pressure was monitored $30 \mathrm{~m}$ above the reference depth. The main test period consists of a producing and non-producing interval as shown in Figs. 11 and 12.

The layering in the reservoir model was chosen in accordance with the layering revealed by seismics and well logging. Three horizontal layers were chosen, layers 1 and 3 being the perforated layers.

An identifiability analysis revealed that it was not possible to determine more than two reservoir parameters, namely the average horizontal permeability $\left(k^{r}\right)$ and the permeability in a thin zone around the well $\left(k^{s}\right)$. Hence, it was not possible to determine the vertical permeability using the data.

Before looking into the estimation problem the problems of noise and model structure validity will be discussed. Analysis of the noise level of the flow-rate and pressure data shows that the predominant noise source stems from the flow-rate measuring device. This noise is filtered to obtain a noise level on the input data which agrees with the actual variations in the flow-rate. A rough estimate of these variations can be obtained by analysing the noise level on the output data and using eqn. (10).

The model which is to be used for parameter estimation, is based on cylindrical coordinates. Parameter estimation runs based on the available data were performed. It was not possible to get a good correspondence between all the data and simulator output. The latter parts of the production and non-production periods could not be reproduced by the simulator. This strongly indicates the influence of effects which are not included in the simulator. These effects, which are most probably due to faults in the reservoir, are placed some distance from the well, and data affected in this way should not be used for optimization. In addition the data in the first part of the production period was not monitored due to debris in the flow from the well (dotted line in Fig. 11).

The above considerations suggest that the times between 9.15 and 11.45 hours, and 18.00 and 22.30 hours should be used for parameter estimation. Since the latter part of the production interval is influenced by effects which are not modeled, special care was taken at the start of the non-producing period. This was done by 


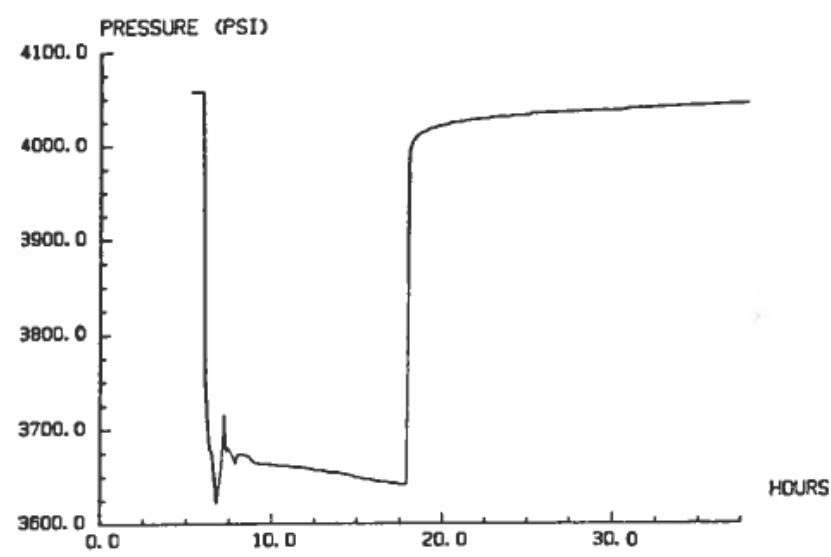

Figure 12. Pressure during the main flow period.

assuming that the boundaries (or faults) discussed above add a constant pressure drop to the well-pressure during the early stages of the non-producing interval in addition to the pressure-drop calculated by the numerical simulator. The bias is estimated in conjucntion with the parameter optimization. This measure compensates for the deficiencies of the model in such a way that the pressure data in the first part of the build-up period can be used for parameter optimization, since the boundaries do not influence the non-producing period in its early stages.

The result of the estimation runs using the limited number of data are given in the following estimates.

$$
k^{r}=438 \mathrm{mD} \quad k^{s}=21.7 \mathrm{mD}
$$

These answers were obtained for large variations in the initial values of the parameter estimates. The simulator output is compared with the real data in Fig. 13.

A $2 \sigma$-confidence interval is calculated using the Hessian-matrix for the estimated permeabilities and an estimate of the noise variance, the result being

$$
k^{r}: 438 \pm 26 \mathrm{mD} \quad k^{s}=21 \cdot 7 \pm 0 \cdot 8 \mathrm{mD}
$$

The information lost by discarding parts of the available data is reasonably small. If it had been possible to utilize all the data, the $2 \sigma$-confidence interval of $k^{r}$ would be reduced from $26 \mathrm{mD}$ to $20 \mathrm{mD}$.

Further analysis of the Hessian matrix shows that the non-producing period contributes most to keeping the confidence intervals small. The reason for this is that the sensitivities regarding the two permeabilities are dynamically heavily correlated during the producing period since the early data (6.00-9.00 hours) of this period cannot be used. This is an important observation, and shows that it is essential that the early time data is of good quality if only the producing interval is to be used for parameter estimation.

The conventional analysis which has been performed (by an oil company) is based on comparing the data with the solution of a homogeneous diffusivity equation by the use of a graphical plot. This is a common analysis technique. Only parts of the data were used in the same manner as above, the permeability was determined to be $420 \mathrm{mD}$, hence within the limits above. The near well permeabilities are difficult to compare, since they are parameterized in different ways. The conventional analysis suggests the presence of boundaries at some distance from the well. 


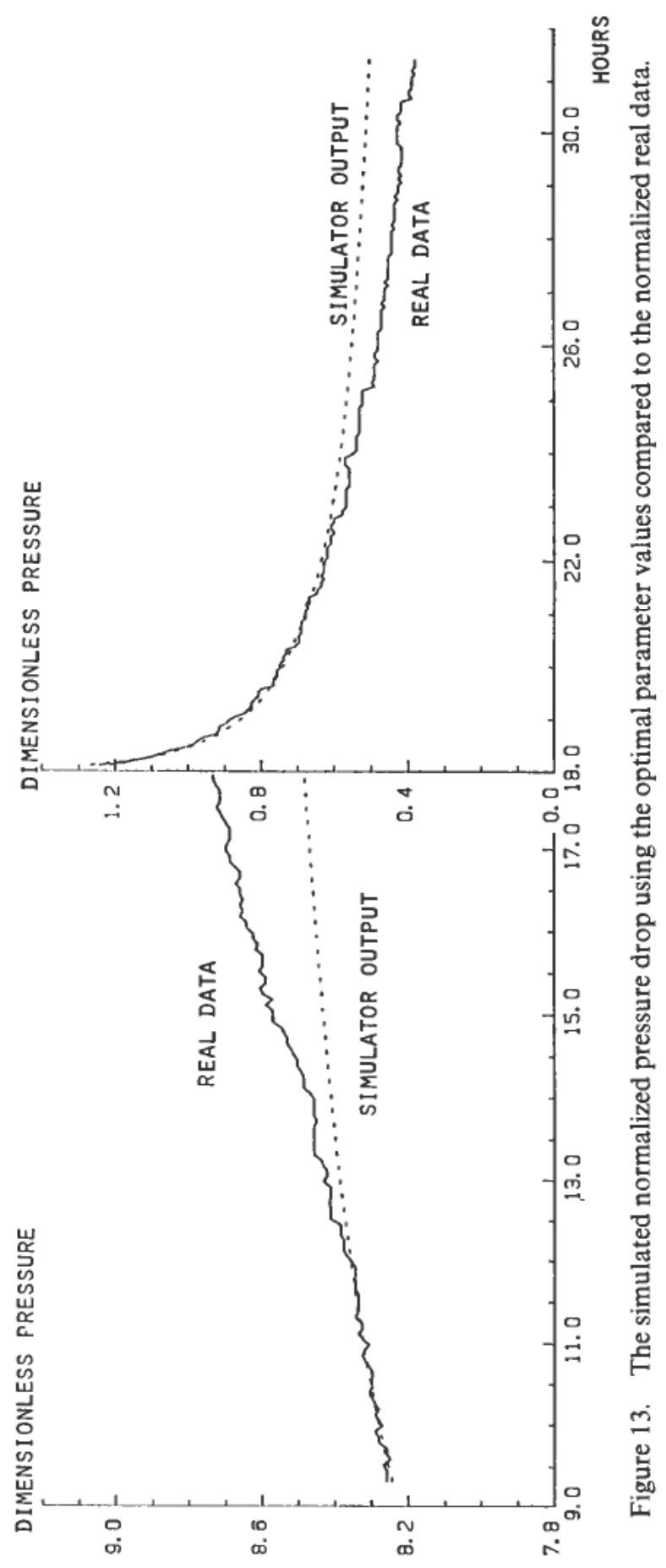


This example highlights the use of error levels for analysing well tests even though the data was not 'rich' enough to fully utilize the model. Two areas, in which more work must be done, are:

Implementation of a more flexible model, for instance by the inclusion of faults.

Data pre-processing, i.e., a mechanism for disregarding data because of model deficiencies or because of large measurement errors.

\section{Discussion}

The two examples in $\S 4$ have illustrated the use of optimal input design and parameter identification in well-testing. It is important to notice that these methods constitute two different ways in which to improve the knowledge of a system.

Optimal input design enhances data quality with respect to certain prespecified unknowns. An analysis of the confidence intervals in Example 1 (\$ 4.1), in addition to other examples regarding both single and multiple well studies presented in Foss (1987(a)), show that on an average, the maximum variance of the parameter estimates decrease by $50 \%$ by the active use of the input design method.

The augmented design criterion is shown to be important in cases where the traditional design criteria exhibit flat minima, and the conditioning of the parameter space is sensitive to the choice of the input sequence.

The use of parameter identification which, as a vital step, includes the choice of parametrization in accordance with error level calculations, improves the analysis of the measured well-test data. Compared to the traditional analysis of well-test data, a quantitative measure of error levels provides a means of balancing the inclusion of prior knowledge versus the choice of the parametrization level, i.e., how many and which parameters may be regarded as unknowns. The unknown parameters may include both variables which describe the basic structure of a model as well as 'normal' parameters (e.g., permeability and porosity). The consequences of discarding data (cf. Example 2, § 4.2) either due to bad data quality or because of the necessity to reduce modeling errors, can also be evaluated.

A shortcoming of the methods presented here is the fact that they are based on a linear analysis. Thus non-linear models will have to be linearized in order to compute the Hessian matrix. This poses no major problem in the case of mild nonlinearities. Severe non-linearities (as is the case in most multi-phase models) do however complicate matters, since a first order linearization only is valid in a limited region about the linearized values. Hence, frequent linearizations are necessary, increasing the computational requirements substantially. In order to assess the methods in these cases, trials must be run using non-linear models.

Modeling errors represent a serious problem which can disrupt any analysis of the kind discussed in this paper. The reason for this is that the analysis, in such a case, is performed on a model which describes a different system from the reservoir in question. There are two measures which should be included, in order to get to grips with this problem.

It is essential to utilize as much as possible of the knowledge which is available prior to the experiment, when deciding on the model.

The calculations should be performed using different parameter values within their probable range of values. In some cases, when the structure of the model 
itself is doubtful (e.g., due to uncertain layering or the possible presence of discontinuities in the layers), different models should be used. In cases with computational limitations, which is the typical case in multiple well studies and history matching, there will be severe limitations on the number of allowable runs.

State estimations has been disregarded in connection with well-testing since a major part of the variations in the flow-rate (which are often large, see Figure 11) couples directly through to the well pressure (cf. eqn. (10)).

\section{NOMENCLATURE}

c isothermal compressibility

$e_{l} \quad$ measurement noise on output no. 1

$g$ input design criterion

$h$ reservoir thickness

$H \quad$ Hessian matrix

$J$ weighted least squares criterion

$k^{r} \quad$ horizontal permeability (*)

$k^{s} \quad$ permeability in a thin zone around the well (*)

$k^{z} \quad$ vertical permeability (*)

$L \quad$ no. of measured outputs

$M \operatorname{dim}(\Theta)$

$N \quad$ no. of time steps

$n r$ no. of grid-blocks in the radial direction

$n z$ no. of grid-blocks in the vertical direction

$p$ pressure

$p_{r j} \quad$ pressure drop between the reservoir $(\mathrm{i}=1)$ and the well in layer $j$

$p_{w} \quad$ well pressure at $z=0$

$q$ flow rate

$r$ radial axis

$r_{e} \quad$ exterior radius of the reservoir model

$r_{w} \quad$ well radius

$t$ time variable

y measured output

$z \quad$ vertical axis

$\Delta z_{j} \quad$ thickness of layer $j$

$\Phi$ porosity

$\mu \quad$ viscosity

$\rho$ density

$\Theta$ unknown parameter vector

$\xi$ weighting factor in the design criterion

$\lambda$ eigenvalue

$\kappa \quad$ condition number

(*) Unit equal to milli-Darcy $\left(\mathrm{mD}=10^{-12} \mathrm{~m}^{2}\right)$

Subscript

$i, j \quad$ radial and vertical index, respectively

Superscript

$n \quad$ time index

- transposed

- estimated value 


\section{REFERENCES}

Azız, K., and Settari, A. (1979). Petroleum Reservoir Simulation. Elsevier Applied Science Publishers, London, UK.

DAKe, L. P. (1978). Fundamentals of Reservoir Engineering. Elsevier Scientific Publishing Company, Amsterdam, The Netherlands.

Foss, B. A. (1987a). On Parameter Identification in Reservoirs. Doctor of Engineering Thesis, The Norwegian Institute of Technology, Trondheim, ISBN 82-7119-005-9.

Foss, B. A. (1987b). A Modified Optimal Input Design Criterion, Proc. 26th IEEE Conference on Decision and Control, Los Angeles, 303-308.

LEE, J. (1982). Well testing. Society of Petroleum Engineers (SPE) Textbook Series, 1, Dallas, USA.

MEHRA, R. M. (1974). Optimal input signals for parameter estimation in dynamic systemssurvey and new results. IEEE Trans. on Automatic Control, vol. no. 19, 753-768.

SILVEY, S. D. (1970). Statistical Interference. Penguin, Baltimore, USA. 